\title{
Using a Slim Function Word Classifier to Recognise Instruction Dialogue Acts
}

\author{
James O’Shea, Zuhair Bandar, Keeley Crockett \\ School of Computing, Mathematics \& Digital Technology, Manchester Metropolitan \\ University, Chester St., Manchester M1 5GD, United Kingdom \\ \{j.d.oshea , z.bandar, k.crockett \}@mmu.ac.uk
}

\begin{abstract}
This paper extends a novel technique for the classification of short texts as Dialogue Acts, based on structural information contained in function words. It investigates the new challenge of discriminating between instructions and a non-instruction mix of questions and statements. The proposed technique extracts features by replacing function words with numeric tokens and replacing each content word with a standard numeric wildcard token. Consequently this is a potentially challenging task for the function-word based approach as the salient feature of an instruction is an imperative verb, which will always be replaced by a wildcard. Nevertheless, the results of the decision tree classifiers produced provide evidence for potentially highly effective classification and they are comparable with initial work on question classification. Improved classification accuracy is expected in future through optimisation of feature extraction.
\end{abstract}

Keywords: Dialogue Act, Speech Act, Classification, Semantic Similarity, Decision Tree.

\section{Introduction}

The initial motivation for this work is the development of a technique for Dialogue Act (DA) classification for use in Conversational Agents (CAs). A CA shares the common properties of intelligent agents in exhibiting autonomy, reactivity and proactiveness [1] and additionally interacts with a human user by means of natural language dialogue [2].

Natural language dialogue involves the exchange of utterances between parties. This work concerns utterances in the form of Short Texts (STs) - which are the length of a typical sentence but do not necessarily obey their grammatical rules. They may arise from entry through a messenger-like text interface or as the output of text-tospeech conversion. 
According to DA theory an utterance can be separated out into its propositional content (what the utterance is about) and its DA, which is the function of the utterance in the conversation. There are several taxonomies which decompose DAs into classes. Prior work has reported using between 2 [3] and 232 [4] classes.

The work of John Searle uses 5 classes: Assertive, Directive, Commissive, Expressive and Declarations [5]. This work also adopts a coarse granularity, but one which is more appropriate for CAs. Specifically, that most dialogue of this type will be composed of questions, instructions and assertions. This assumption is supported by prior work in dialogue systems [6] and robotics [7].

Examples of these DAs are: "Is the door shut?" (question), "The door is shut" (assertion) and "Shut the door!" (instruction). All are derived from the propositional content "door is shut." Each of the sentences contains the noun door and the verb shut. The remaining words, the and is are function words.

Prior work has produced a Slim Function Word Classifier (SFWC) which discriminates effectively between question and non-question DAs. The research question addressed by this study is "Can an SFWC be trained to discriminate between instruction and non-instruction DAs?" This leads to the hypotheses:

H1: An SFWC scores significantly higher than chance in discriminating between Instruction and Non-Instruction DAs.

H0: An SFWC scores no higher than chance in discriminating between Instruction and Non-Instruction DAs.

The level of classification accuracy which would be obtained by chance for the dataset used in this study is $50 \%$. Hypothesis testing uses the corrected re-sampled $t$ test to determine whether the classification accuracy is significantly different from this baseline.

The rest of this paper is concerned with testing these Hypotheses. Section 1 briefly reviews prior work on features for DA classification, function words and choice of classifier. Section 2 describes the experimental procedure including creation of the DA dataset and experiments to train and test classifiers. Section 3 discusses the results and Section 4 the conclusions and recommendations for future work.

\subsection{Extraction of Features for DA Classifiers}

There are a number of requirements of the features extracted from utterances for DA classification. First, they should actually encode sufficient discriminating information to support accurate classification. Second, they should be capable of being extracted by computationally efficient algorithms. Third, they should be robust to the variations in real-world data.

The most commonly used feature is the n-gram, a short sequence of contiguous words. N-grams are created by extracting them from a real-world corpus [8,9]. Any realistically sized corpus will generate large numbers of candidates. Consequently the set of potential n-grams is analysed to find a subset which are good predictors for the classification task. For example, [9] used a set of 100 logs from a social interaction computer game containing 4,295 utterances with an average length of 4 words as a 
training set, using a mutual information measure to find the best 50 predictive unigrams, bigrams and trigrams for 9 distinct DAs.

Cue phrases are another type of feature, similar to n-grams, which have been used for DA classification. However, a cue phrase is used in the classifier to predict a single DA class $[8,10]$. During training, a cue phrase is selected and assigned the single DA class for which it has the highest predictivity value. During classification if there are two or more cue phrases with the same predictivity the cue phrase with the highest frequency of occurrence is used, potentially discarding valuable classification information.

Latent Semantic Analysis (LSA) has also been adapted for DA classification [4]. LSA uses terms organised into vectors and the measurement is made between the vectors in a semantic space. LSA is an information retrieval system, so the input text corresponds to an LSA "query" and the DA is that of the closest matching "document". The LSA semantic spaces are constructed from various corpora, for example general reading up to $1^{\text {st }}$ year college. Normally LSA completely discards function words in forming its vectors [11] although this is not clarified in [4].

Some complex combinations of features have been used for DA classification. Keizer et al [3] used length, various starting bigrams, presence of particular words, positive/negative references and presence of particular grammatical classes to classify utterances into two different classes: forward-looking and backward-looking. Forward-looking functions have an effect on following dialogue and backwardlooking functions relate to previous dialogue. Verbree et al [12] used a combination of features including the presence of a question mark, a count of the occurrences of the word OR, utterance length, a bigram of the labels of the last two DAs, n-grams of pos-tags for the utterance, n-grams of words in the utterance and the top 10 predictive individual words.

O'Shea et al [13] took a radically different approach to classifying the question DA (vs. non-questions) by using function words only. This method replaced function words by unique tokens and every content word by a common wildcard token.

Performance is difficult to compare, between studies, because there is no established benchmark in this field. Keizer achieved classification accuracy of $76.5 \%$ - $88.2 \%$ on the SCHISMA corpus. Verbree achieved $59.76 \%-89.27 \%$, on the ICSI, Switchboard and AMI corpora. O'Shea achieved an accuracy of $89.43 \%$ using a data set collected for the study which was representative of dialogue conducted via the web. Regardless of relative performance, the features used in the work of Keizer and Verbree are complex and computationally intensive to extract, apart from Cue Phrases which may discard considerable useful information.

\subsection{Function Words}

English words can be partitioned into two classes: Function Words (e.g. articles, prepositions, determiners etc.) and Content Words (nouns, verbs, adjectives and adverbs). An important difference between them is that Function Words are a closed class (the English Language has evolved all of the Function words that it needs and no new words are added to the class). Conversely, new Content words emerge 
continuously - for example the verb "to tweet" (in the sense of posting a message on Twitter).

There is no definitive list of function words, but they can be extracted from "Stop word" lists which contain mostly function words with a few high frequency content words mixed in. Identifying stop words is important in information retrieval applications where they are removed because they make little or no distinction between documents [11]. One exception is the STASIS measure [14] which recognises the importance of function words because they carry structural information which is useful in interpreting sentence meaning. Again, there is no definitive list of stop words, although one by van Rijsbergen [15], which is cited frequently contains 250 words. The set of 264 function words compiled for the SFWC [13] is used in this paper. Function words are potentially good features for classification of DAs because they are easily identifiable using small lookup tables, they are stable over time and they are used in all domains so should generalise well.

\subsection{Choice of Classifier}

A representative range of classifiers has been used in DA classification including Decision Trees [12] Naïve Bayes [3], the Bayesian Network [16] and Artificial Neural Networks [17]. The initial work on the SFWC provided evidence that Decision Trees (DTs) were an effective form of classifier to use with function word features [13] and consequently DTs were chosen for this work.

One of the most well-established DT algorithms is C4.5 [18]. DTs partition the sample space recursively and the outcome is a set of rules induced from a training set of example instances previously labelled with their classes.

The starting point of a DT is one node with all examples labelled as belonging to a single class. In realistic examples this node is "impure" i.e. the examples from the training set contain more than one class. During training, impure nodes are successively partitioned to create new, purer nodes. The final, leaf, nodes are labelled with the appropriate class.

Impure leaves may occur if the attributes have been tested exhaustively without reaching purity. These are labelled with the majority class; consequently test cases from the minority class which reach the leaf during classification result in errors.

There is also the possibility that a tree may model the training data at too high a level of detail, in which case it will not generalise effectively when used with other data. This is prevented by pruning the tree. Confidence Level pruning [18] decides on the level of pruning based on an estimate (derived from the training data) of the likely level of error with other data. Minimum Number of Objects (MNO) pruning [19] removes leaves with less than a specified number of cases and re-distributes them amongst leaves at the level above in the tree.

DTs have two distinct advantages over other classifiers. The first is that the rules "explain" how they reach their decisions very clearly and (combined with pruning) provide a greater insight into the problem domain. The second is that the rules generated by a decision tree can be converted into highly efficient code quite easily for implementation of real-time systems. 


\section{Experimental Procedure}

\subsection{Creation of the Dataset}

Investigation of the hypothesis that a SFWC can discriminate effectively between Instruction and Non-Instruction DAs requires a suitable tagged (classified) dataset for training and testing the classifier.

There is no standard dataset and those used in studies vary widely using different numbers of DA classes (from 2 [3] to 232 different tags [4]), sizes (81 utterances [3] to 223,000 utterances [8]) and composition (e.g. one dataset of 2794 DAs was drawn from only two humans [20]). A moderately sized dataset can be used in distinguishing a single DA class from the rest (600 questions and 600 non-questions) [13]. Using 60fold cross validation with this dataset resulted in over 1000 cases being used for training, in line with a rule-of-thumb suggested by Quinlan [18].

This study uses the questions and non-questions from [13] as the source of noninstructions to be discriminated against. 25 different D.I.Y and self-help sites provided useful sources of instructions, e.g. "Central heating project" and "How to Bisque fire pottery." The instructions were largely consistent with spoken dialogue.

The initial assumption was that even straightforward instructions would prove difficult to classify because their most salient feature, an imperative verb, is converted to a wildcard. In fact most of the questions and statements begin with a function word so this difference in itself provided information for classification.

This was a similar position to that of the straightforward questions vs. nonquestions in [13] where the straightforward questions started with auxiliary verbs or wh-chefts. A similar procedure to that in [13] was adopted to increase the challenge. In this case a set of 1-word preambles was used to push the imperative verb into the second position where the non-instructions were more likely to have a wildcard token and the function words would play a greater role in inferring the occurrence of an instruction. All of the 1-word preambles (e.g. "Again", "Actually") were semantically feasible components of instructions, collected from the same sources as the dataset. The outcome was that a set comprised of 50\% (600) instructions with 1-word preambles, 25\% (300) straightforward questions and 25\% (300) straightforward statements was produced to train and test the instruction / non-instruction classifier (examples are shown in table 1).

Table 1. Example training data

\begin{tabular}{|l|l|}
\hline Category & Example \\
\hline FAQ question & $\begin{array}{l}\text { Are the Canada pension plan and Canadian old age } \\
\text { security benefits taxable? }\end{array}$ \\
\hline Blog non-question & $\begin{array}{l}\text { She's an insanely talented, sought after calligrapher } \\
\text { and one of our dearest long-time friends. }\end{array}$ \\
\hline $\begin{array}{l}\text { Straightforward } \\
\text { Instruction }\end{array}$ & $\begin{array}{l}\text { Do as advised in the handbook where the handbook } \\
\text { and our advice appear in conflict. }\end{array}$ \\
\hline Instruction with preamble & $\begin{array}{l}\text { Again do as advised in the handbook where the } \\
\text { handbook and our advice appear in conflict. }\end{array}$ \\
\hline
\end{tabular}


For each category, all of the available sentences were preprocessed to restrict the length to a maximum of 25 words, expand contracted forms, tokenise them to extract features for DT construction and remove duplicates occurring due to loss of information in the tokenising process. This was followed by random selection of the number required for each class in the dataset. Decision trees require fixed length records, so an upper limit of 25 words was set (determined empirically based on prior experience of creating real-world CAs).

\subsection{Preprocessing the data}

Auxiliary verbs such as do, would and can are function words which play an important role in constructing tenses and expressing conditions such as permission and ability. These words take part in contractions such as shouldn't, can't and I'd. To ensure that these forms were tokenised correctly, the contractions were replaced with their full forms, e.g. should not, can not before tokenisation. There may be some ambiguity and a brute force solution (e.g. replacing all I'd forms with I would) has been taken. This approach avoids a potentially computationally intensive parsing stage and did not have a noticeable impact on performance in [13]. All punctuation, apart from apostrophes used in the possessive form, was then stripped out. As the possessive form is a noun variant this is permissible. One consequence is that the presence of a question mark (as used in [12]) is not currently used in this technique.

The preprocessed STs were then tokenised, word-by-word. Each word was looked up in a table of function words which also contained a unique numeric token (range 1264) for each function word. If there was a match the word was replaced by the relevant token. If the word was not found in the function word table it was assumed to be a content word and replaced by the token 0 . If the sentence contained less than 25 words, it was padded out to a length of 25 tokens by inserting "no word present" tokens with a value of 300 . Thus the classifier could distinguish very clearly between specific function words, content words in general and empty spaces at the end of sentences in partitioning the problem space. An example of a tokenised instruction is shown in table 2 .

Table 2. Tokenisation of training data

\begin{tabular}{|l|l|}
\hline Question & $\begin{array}{l}\text { Take your multimeter probes and connect them to the } \\
\text { outer prongs of the stove's power cord next. }\end{array}$ \\
\hline $\begin{array}{l}\text { Tokenised } \\
\text { form }\end{array}$ & $\begin{array}{l}0,261,0,0,22,0,192,212,190,0,0,148,190,0,0,0,138, \\
300,300,300,300,300,300,300,300\end{array}$ \\
\hline
\end{tabular}

The function word table used in this paper contains the words sorted in alphabetical order, so that 1 corresponds to "a" and 264 corresponds to "yourselves." In the example, the verb "Take" is replaced by 0 because it is a content word. "your" is tokenised as 261 , "multimeter probes" is composed of two content words and each is replaced by 0 . "And" is replaced by 22 , "them" by 192 , "to" by 212 , "the" by 190 , "of" by 148 and "next" by 138. The other content words are replaced by 0s in the same way as "Take", "multimeter" and "probes." 


\subsection{Decision Tree Experimental Trials}

This section evaluates the performance of a C4.5 decision tree classifier trained on the dataset (using cross validation) using a range of pruning parameters to identify the best Classification Accuracy and the most compact DTs that can be obtained. Both Confidence Interval (Conf) and Minimum Number of Objects (MNO) were used as pruning techniques. All of the experiments reported in this paper used 60-fold cross validation and were conducted using the Weka data mining package.

The DTs were constructed using the C4.5 version 8 algorithm known as J48 in Weka. Weka also reports significant differences between results in a series against a baseline, using the corrected re-sampled $t$-test with $\alpha=0.05$. In the following experiments the baseline was the CA achieved by a C4.5 DT using pruning values of Conf $=0.25$ and $\mathrm{MNO}=2$.

\subsubsection{Experiments $1 \& 2$}

Experiment 1 examined the effect of pruning at a series of confidence levels running from the default value of 0.25 to 0.05 . The first entry, Conf $=0.25(\mathrm{MNO}=2)$ sets the baseline for comparison of the other entries. Table 3 shows that increased pruning leads to increasing CA (statistically significant at confidence $=0.15$ ). This reaches a maximum of 89.23 at confidence 0.0775 (39 - 91 nodes).

Table 3. Results of pruning in experiment 1

\begin{tabular}{|l|l|l|l|l|l|}
\hline Conf & 0.25 & 0.2 & 0.15 & 0.1 & 0.05 \\
\hline Classification Accuracy & 87.98 & 88.22 & 88.87 & 88.98 & 88.63 \\
\hline Tree Size & $63-121$ & $59-109$ & $51-103$ & $45-97$ & $23-79$ \\
\hline
\end{tabular}

Experiment 2 examined the effect of confidence pruning to produce the most compact, efficient tree that did not suffer a statistically significant loss of CA.

Experiment 1 was repeated, using pruning confidence intervals running from $1.0 \mathrm{E}-3$ to $1.0 \mathrm{E}-5$. Table 4 shows the results.

Table 4. Results of pruning in experiment 2

\begin{tabular}{|l|l|l|l|l|l|}
\hline Conf & $1.0 \mathrm{E}-3$ & $5.0 \mathrm{E}-4$ & $1.0 \mathrm{E}-4$ & $5.0 \mathrm{E}-5$ & $1.0 \mathrm{E}-5$ \\
\hline Classification Accuracy & 87.92 & 87.92 & 87.90 & 87.89 & 87.87 \\
\hline Tree Size & $19-27$ & $19-27$ & $19-23$ & $19-23$ & $15-23$ \\
\hline
\end{tabular}

Execution was interrupted at the 5.0E-6 confidence level, presumably due to a floating-point arithmetic error. This was before a significant drop in CA below the baseline was reached. 


\subsubsection{Experiments $3 \& 4$}

Experiment 3 examined the effect of pruning at a series of MNO levels running from the default value of 2 to 20 (table 5). This pruning did not discover a significant change in CA. The maximum CA obtained with MNO pruning was $88.03 \%$ at MNO $=4$, which was not significantly better than the baseline value.

Table 5. Results of pruning in experiment 3

\begin{tabular}{|l|l|l|l|l|l|}
\hline MNO & 2 & 5 & 10 & 15 & 20 \\
\hline Classification Accuracy & 87.98 & 87.68 & 86.98 & 87.03 & 86.91 \\
\hline Tree Size & $63-121$ & $39-91$ & $27-53$ & $19-33$ & $15-29$ \\
\hline
\end{tabular}

Experiment 4 examined the effect of MNO pruning to produce the most compact, efficient tree that did not suffer a statistically significant loss of CA.

Table 6. Results of pruning in experiment 4

\begin{tabular}{|l|l|l|l|l|l|}
\hline MNO & 36 & 37 & 38 & 39 & 40 \\
\hline Classification Accuracy & 87.29 & 87.19 & 86.98 & 86.43 & 85.49 \\
\hline Tree Size & $11-21$ & $11-21$ & $11-21$ & $7-21$ & $7-19$ \\
\hline
\end{tabular}

Table 6 shows the results of pruning using MNO levels running from 36 to 40 instances. At $\mathrm{MNO}=40$ the reduction in $\mathrm{CA}$ from the baseline was significant. Pruning at $\mathrm{MNO}=39$ gave a good combination of tree size and $\mathrm{CA}$.

\section{Discussion of Results}

Confidence level pruning achieved a CA of $89.23 \%$ at confidence $=0.0775$ and $\mathrm{MNO}$ pruning achieved a $\mathrm{CA}$ of $88.03 \%$ at $\mathrm{MNO}=4$. The average baseline tree size was 93 nodes. It was not possible to discover the optimum pruning level with confidencebased pruning, but pruning at $\mathrm{MNO}=39$ produced an average tree size of 15 . The maximum depth of nesting of this tree was 5, suggesting that efficient implementation would have a low computational overhead.

\section{Conclusions and Future Work}

The results of the experiments provide good evidence to support the alternative hypothesis that an SFWC scores significantly higher than chance in discriminating between Instruction and Non-Instruction DAs. Statistically significant results (using the corrected re-sampled $t$-test) for CA well above baseline have been obtained. The reduction in tree size from pruning provides further strong evidence that true generalisation is taking place. 
The pruned decision trees combine function word features ranging from the $1^{\text {st }}$ to the $6^{\text {th }}$ word. This indicates that the technique is exploiting more than simple n-gram information and that the function words in the vicinity of the imperative verb are key features in classification.

When these results are considered with those from [13], good evidence is accumulating to support the general principle of using function words for DA classification.

Future work will involve the development of specialist classifiers for particular variants of the instruction form, which could be combined into a single multiclassifier. It will also investigate the possibility of producing a gold standard benchmark dataset from the DA training and testing datasets produced. Additionally, experiments will be conducted on optimising the encoding of features. This is expected to reduce top level splitting and preserve information for better splitting at later stages in the tree. It is also expected to improve generalisation by allowing a particular route through the tree to represent more cases.

\section{References}

1. Wooldridge, M. and Jennings, N.R.: Intelligent Agents: theory and practice. The Knowledge Engineering Review, 10(2), 115-152 (1995)

2. Bickmore, T. and Cassell, J.: 'How about this weather?' Social Dialog with Embodied Conversational Agents. In: The American Association for Artificial Intelligence (AAAI) Fall Symposium on "Narrative Intelligence". Cape Cod, MA (2000)

3. Keizer, S., op den Akker, R. and Nijholt, A.: Dialogue Act Recognition with Bayesian Networks for Dutch Dialogues. In: Third SIGdial Workshop on Discourse and Dialogue. Philadelphia (2002)

4. Serafin, R., Di Eugenio, B. and Glass M.: Latent Semantic Analysis for dialogue act classification. In: The 2003 Conference of the North American Chapter of the Association for Computational Linguistics on Human Language Technology. Edmonton, Canada (2003)

5. Searle, J.R.: Mind, Language and Society. Weidenfield \& Nicholson (1999)

6. Crockett, K., et al. Bullying and Debt: Developing Novel Applications of Dialogue Systems. In: Knowledge and Reasoning in Practical Dialogue Systems (IJCAI). Pasadena, CA: IJCAI (2009)

7. Längle, T., et al.: KANTRA - A Natural Language Interface for Intelligent Robots. In: Intelligent Autonomous Systems (IAS 4). Amsterdam (1995)

8. Webb, N., Hepple, M. and Wilks Y.: Dialogue Act Classification Based on Intra-Utterance Features. In: AAAI 2005. Pittsburgh, Pennsylvania: AAAI Press (2005)

9. Orkin, J. and Roy D.: Semi-Automated Dialogue Act Classification for Situated Social Agents in Games. In: The AAMAS Agents for Games \& Simulations Workshop. (2010) 
10. Webb, N. and Liu T.: Investigating the Portability of Corpus-Derived Cue Phrases for Dialogue Act Classification. In: Proceedings of the 22nd International Conference on Computational Linguistics (Coling 2008). Manchester (2008)

11. Deerwester, S., et al.: Indexing by Latent Semantic Analysis. Journal of the American Society of Information Science, 41(6), 391-407 (1990)

12. Verbree, D., Rienks, R. and Heylen D.: Dialogue-Act Tagging Using Smart Feature Selection; Results On Multiple Corpora. in IEEE Spoken Language Technology Workshop. (2006)

13. O'Shea, J., Bandar, Z. and Crockett, K.: A Machine Learning Approach to Speech Act Classification Using Function Words. In Jedrzejowicz, P., Nguyen, N. T., Howlett, R. J., Jain, L.C. (eds.) KES-AMSTA 2010. LNAI, vol 6071, pp. 82-91 Springer, Heidelberg (2010)

14. Li, Y., et al.: Sentence Similarity Based on Semantic Nets and Corpus Statistics. IEEE Transactions on Knowledge and Data Engineering, 18(8), 1138-1150 (2006)

15. van Rijsbergen, C.J.: Information Retrieval. Boston: Butterworths (1980)

16. Stolcke, A., et al.: Dialogue Act Modeling for Automatic Tagging and Recognition of Conversational Speech. Computational Linguistics 26, 339373 (2000)

17. Kim, K., Kim, H. and Seo, J.: A neural network model with feature selection for Korean speech act classification. Int. J. Neural Syst., 14(6), 407-414 (2004)

18. Quinlan, J.R.: C4.5: programs for machine learning. Morgan Kaufmann series in machine learning. San Mateo, California: Morgan Kaufmann Publishers (1993)

19. Witten, I.H. and Eibe, F.: Data Mining: Practical Machine Learning Tools and Techniques. 2 ed. San Francisco: Elsevier (2005)

20. Venkataraman, A., Stolcke, A. and Shriberg, E.: Automatic Dialog Act Labeling With Minimal Supervision. In: 9th Australian International Conference on Speech Science and Technology. (2002) 